\title{
Motion aftereffect with subjective contours
}

\author{
ANDREW T. SMITH and RAY OVER \\ University of Queensland, St. Lucia, Queensland, Australia 4067
}

\begin{abstract}
Stationary lines appear to move from left to right following exposure to lines moving from right to left. This aftereffect, which normally is generated by exposure to moving edges that are defined in terms of local luminance discontinuity, can also be induced by adaptation to displays containing subjective contours. In both cases, stereodeficient observers demonstrated reduced interocular transfer of the aftereffect relative to stereonormal observers. Since interocular transfer of the motion aftereffect entails binocular function within the visual system, these results suggest that the perception of subjective contours depends on excitation of neural feature detectors rather than simply on cognitive inference.
\end{abstract}

A stationary display viewed following exposure to a pattern moving in one direction will appear to move slowly in the opposite direction. Following Barlow and Hill (1963) and Sutherland (1961), this aftereffect has been attributed to selective adaptation of directionally sensitive motion analyzers within the visual system. The proposition is that cells excited by motion in the direction of the inspection pattern are inactive when this motion ceases, whereas cells sensitive to the opposite direction of motion remain spontaneously active. This imbalance in neural activity occurring through postexcitatory suppression results in the stationary test display yielding the pattern of neural activity that would normally be generated by a pattern moving slowly in the direction opposite to inspection. Evidence that there are directionally sensitive motion detectors in the human visual system has come from evoked potential measures (Clarke, 1973) and psychophysical masking functions (Sekuler \& Ganz, 1963).

The motion aftereffect has been studied in detail using stimuli in which all the perceived edges within the display are correlated with spatial discontinuity in luminance. The present study measures the motion aftereffect induced by exposure to displays in which edges are visible at local sites where the stimulus is homogeneous (such as Figure 1A). These "anomalous" contour effects, first described by Schumann (1904), have been variously called subjective contours, cognitive contours, virtual contours, illusory contours, contours without gradients, and quasiperceptual boundaries on the grounds that at a punctate level there is no physical stimulus for the edges that are visible.

This research was reported in a dissertation submitted in 1976 by the first author in partial fulfillment of the degree of Master of Science at the University of Queensland. The first author is now in the Department of Communication and Neurosciences, University of Keele, Keele, Staffordshire ST5 5BG, England, while the second author (to whom requests for reprints should be sent) is now at the Department of Psychology, La Trobe University, Bundoora, Australia 3083.

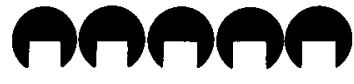

A

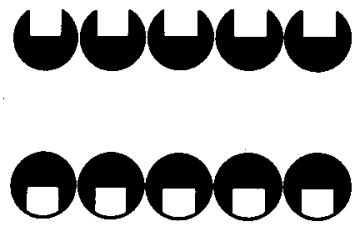

$\mathbf{B}$

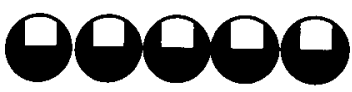

Figure 1. Subjective contour pattern (A) and control stimulus (B).

Within one tradition (e.g., Coren, 1972; Gregory, 1972), the perception of subjective contours is attributed to faulty cognitive inference. However, Ginsburg (1975) has argued that there is a stimulus correlate for so-called "anomalous"' edge perception when the visual display yielding the effect is considered in terms of its Fourier spectrum rather than by reference to local geometry. On such grounds, it is not surprising that orientation masking as well as tilt aftereffects and illusions can be induced with subjective contours as readily as with edges defined in terms of local luminance discontinuity (Smith \& Over, 1975, 1977). These latter effects traditionally are explained by reference to adaptation or inhibition of orientationsensitive detectors in the visual system (see Over, 1971).

Tynan and Sekuler (1975) noted that "phantom" stripes appear to move through a physically homogeneous region of the visual field when vertical stripes move above or below these regions. Although the basis for these perceived lines may be the same as that for subjective contours in general, it is of interest to note that "phantom" lines were visible when the surround gratings were moving, but not when they were stationary. Further, "phantom" 
lines moving in opposite directions were apparent when the top and bottom gratings moved in opposite directions. Weisstein, Maguire, and Berbaum (1977) have shown that exposure to moving "phantom" stripes generates a motion aftereffect of the same strength as that found after adaptation to moving edges defined in terms of luminance discontinuity. The present study measured the perceived movement of a stationary grating presented, following exposure to Figure 1A, in the region of the visual field in which moving subjective contours had been perceived. Interocular transfer was studied with observers of differing stereoacuity in order to establish the similarity of the aftereffect induced with subjective contours to the classical motion aftereffect.

The motion aftereffect induced by exposure to lines correlated with local luminance discontinuity demonstrates interocular transfer, although the size of the aftereffect is greater when the moving (inspection) and stationary (test) patterns are viewed by the same eye than when they are seen by different eyes (Mitchell, Reardon, \& Muir, 1975). Binocular neural function is a necessary condition for interocular. transfer of a spatial aftereffect; the same detectors that were adapted with presentation of the inspection stimulus to one eye must be engaged in an analysis of the test stimulus shown to the other eye for interocular transfer to occur. Binocular visual function is impaired if the normal coordinated function of the two eyes is interfered with in the first few years of life; adults with a history of strabismus or anismetropia in childhood are stereodeficient or stereoblind, and they have limited (if any) ability to judge depth solely on the basis of binocular disparity information. These same people exhibit little or no interocular transfer of spatial aftereffects, although aftereffects of normal size are found when the inspection and test stimuli are viewed by the same eye (Mitchell et al., 1975; Mitchell \& Ware, 1974; Movshon, Chambers, \& Blakemore, 1972).

It is demonstrated in the present study that the motion aftereffect can be induced by exposure to moving edges independently of whether the edges are subjective contours or real contours (correlated with local luminance discontinuity). With both types of display, the extent of interocular transfer of the motion aftereffect is related to an observer's stereoacuity.

\section{METHOD}

Undergraduate students were screened with reference to spatial acuity in each eye (Snellen test) and their ability to perceive depth on the Titmus stereo-test and on Julesz random-dot stereopatterns (see Julesz, 1971, p. 270). The 10 stereonormal observers used in the present study had Snellen acuity of $6 / 20$ or better in each eye, and could discriminate depth on the Titmus test on the basis of a disparityof 40 secarc or less and on the
Julesz test with displays containing $\mathbf{2 0 \%}$ or more complementation (see Julesz, 1971, p. 270). The 6 stereodeficient observers had Snellen acuity of $6 / 20$ or better in each eye, but they were unable to perceive depth on the Titmus test on the basis of a disparity less than 200 secarc or with any of the Julesz patterns.

The experiment measured the extent to which a stationary test grating defined by real contours appeared to move following exposure to a moving inspection grating of either real or subjective contours. The subjective contour display consisted of a series of vertical bars, each subtending $5^{\circ} 30^{\prime}$ by $1^{\circ} 30^{\prime}$ and drawn side by side to form a grating having a spatial frequency of .38 cycles $/^{\circ}$ (see Figure 1A). These contours were shown on a continuous belt of white card which was moved mechanically from right to left at $1.4 \mathrm{cycles} / \mathrm{sec}$ behind a rectangular aperture subtending $13^{\circ}$ horizontally and $10^{\circ}$ vertically. The real contour display was produced by attaching strips of white tape of the same dimensions as the subjective contours to white card. The tape and the white card differed slightly in luminance to approximate the "whiter than white" phenomenal impression of the subjective contour. To make the subjective and real contour displays otherwise equivalent, additional contour information of the type shown in Figure 1B was positioned on the white card at the ends of each strip of tape. For both real and subjective contours, the luminance of the bar zone was $8.6 \mathrm{~cd} / \mathrm{m}^{2}$. The stationary test grating that was viewed at the end of the inspection period consisted of white bars (contrast .5 , space-average luminance $2.2 \mathrm{~cd} / \mathrm{m}^{2}$, spatial frequency .38 cycles $/{ }^{\circ}$ ) on a black ground. These bars subtended $1^{\circ} 10^{\prime}$ in height.

Attention needs to be given to the possibility that any motion aftereffect consequent upon inspection of Figure $1 \mathrm{~A}$ is under the control of local luminance discontinuities present within this display, rather than being due to the subjective contours themselves. These two variables cannot be studied in isolation, since a display must contain real-contour information in order that subjective contours be induced. Observers were required to maintain fixation at the center of the stimulus during both inspection and test stages; the test grating thus fell within the part of the visual field in which the subjective contours were seen, but did not overlap the adjacent real-contour information. In addition, a control condition was employed, with the motion aftereffect being measured following exposure to an inspection stimulus (Figure 1B) that did not yield subjective contours although it contained all the real-contour information that was present in Figure 1A.

The aftereffect was measured by a manual tracking technique; observers moved a pen on a carriageway (outside the field of view) at the speed and in the direction in which the lines in the test display appeared to be moving. The pen left a trace on recording paper that moved at $.25 \mathrm{~cm} / \mathrm{sec}$ at right angles to the direction of the carriageway, and the size of the aftereffect was given by the slope of the trace. This measurement technique, which has been employed in several aftereffect studies, has a test-retest reliability of +.83 , and when it was calibrated by requiring observers to move the pen at the same apparent speed as a grating moving at known velocity, the data were fitted by the function $\mathrm{y}=.06 \mathrm{x}$, where $\mathrm{y}$ is the mean pen displacement in millimeters and $\mathrm{x}$ is stimulus velocity in degrees per second (Over \& Lovegrove, 1973).

Polarizing filters were used so that the inspection and test patterns could be viewed by the same eye (monocular condition) or the inspection pattern by one eye and the test pattern by the other eye (interocular transfer condition). Three measures of the aftereffect were obtained from each observer under each condition following exposure to real contours, subjective contours, and the control stimulus. In each case, there was an inspection period lasting $90 \mathrm{sec}$ before the observer tracked the apparent motion of the test stimulus of a 10-sec interval; the moving inspection pattern was then viewed for two further periods of $15 \mathrm{sec}$, each followed by $10 \mathrm{sec}$ tracking of the test stimulus. 


\section{RESULTS}

Because observers differed in the overall extent of pen movement, raw scores were converted to percentages. For each subject, the three measures obtained under a given condition were added, and the largest value obtained under the six conditions was given a value of 1.0. The aftereffects obtained under the other five conditions were expressed as a proportion of this value. The mean aftereffects obtained for the three inspection displays, the two viewing conditions, and as a function of the stereoscopic competence of the observers are shown in Figure 2. Standard errors ranged from .06 to .09 for stereonormal observers and from .05 to .09 for stereodeficient observers. For both stereonormal observers $[F(2,18)$ $=18.21, \mathrm{p}<.01]$ and stereodeficient observers $[F(2,10)=7.99, p<.01]$, the mean aftereffect varied as a function of the inspection stimulus. Multiple comparisons by a Newman-Keuls test showed that within both groups, adaptation to the control stimulus resulted in a smaller aftereffect than was found after exposure to either subjective or real contours; the latter inspection stimuli yielded similar amounts of motion aftereffect. Thus, although part of the aftereffect induced by exposure to Figure 1A is clearly dependent on the local luminance discontinuities present within the display, a significant component can be attributed to the subject contours themselves.

The aftereffect was smaller under conditions of interocular transfer than with monocular viewing $[F(1,14)=38.65, p<.01]$ : The interaction between viewing condition and the stereoscopic competence of the observers was also significant $[\mathrm{F}(1,14)=5.21$, $\mathrm{p}<.05$ ]. Multiple comparisons showed that, with monocular viewing, the magnitude of aftereffect was similar for stereonormal and stereodeficient observ-

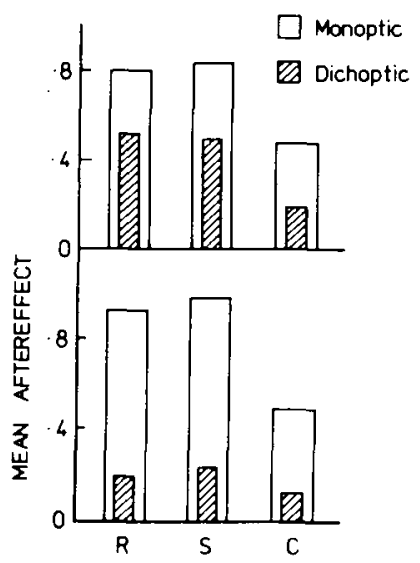

Figure 2. Mean percentage of maximum aftereffect obtained for stereonormal (top) and stereodeficient (bottom) observers for the three inspection stimuli (real contours, subjective contours, control) under monoptic and dichoptic conditions. vers. However, the stereonormal observers showed a significantly higher level of interocular transfer (on average, $58 \%$ of the aftereffect found with monocular viewing) than the stereodeficient observers (average interocular transfer of $21 \%$ ).

\section{DISCUSSION}

The results show that the motion aftereffect can be induced by exposure to moving subjective contours as readily as by inspection of contours defined in terms of luminance discontinuity. This finding matches the results obtained by Weisstein, Maguire, and Berbaum (1977), and it also parallels the relationships between subjective and real contours reported by Smith and Over $(1975,1977)$ for orientation-selective masking, aftereffect, and illusion. Although such data suggest that it is inappropriate to attribute the perception of subjective and real contours to basically different mechanisms, they do not allow direct identification of the operations that underlie the perception of subjective contours. For example, although most accounts attribute spatial aftereffects to processes such as postexcitatory suppression, several explanations, such as normalization theory (Gibson, 1937), could be applied to aftereffects obtained with real and subjective contours without reference to operations within the visual system.

It is difficult, however, to explain the relationship between stereoscopic competence and interocular transfer of spatial aftereffects without direct reference to the operating characteristics of the visual system. Adaptation theories attribute aftereffects to postexcitatory suppression of visual feature detectors, and in such terms, interocular transfer would occur to the extent that the detectors normally engaged in signaling the test stimulus are in a nonresponsive state following prior stimulation through the other eye. Binocular function is thus a necessary condition for interocular transfer. Binocular responsiveness is lost, and stereoacuity is impaired, under certain conditions of abnormal visual stimulation in early experience; this same factor lowers interocular transfer of spatial aftereffects (Banks, Aslin, \& Letson, 1975; Mitchell \& Ware, 1974; Mitchell et al., 1975, Movshon et al., 1972). Stereodeficient subjects demonstrate the same level of aftereffect under monocular viewing conditions as observers with normal stereoacuity; the failure of the aftereffect to exhibit interocular transfer must be attributed to lack of binocular spatial detectors within the visual system. This interpretation can be applied to the results of the present study by supposing that common neural mechanisms underlie the perception of subjective contours and the perception of edges correlated with luminance discontinuity. 


\section{REFERENCES}

Banks, M. S., Aslin, R. N., \& Letson, R. D. Sensitive period for the development of human binocular vision. Science, 1975, 190, 675-677.

BaRLow, H. B., \& HrLl, R. M. Evidence for a physiological explanation of the waterfall phenomenon and figural aftereffects. Nature, 1963, 200, 1345-1347.

Clarke, P. G. H. Visual evoked potentials to changes in the motion of a patterned field. Experimental Brain Research, 1973. 18, 145-155.

Coren, S. Subjective contours and apparent depth. Psychological Review, 1972, 79, 359-367.

GrBson, J. J. Adaptation with negative aftereffect. Psychological Review, 1937, 44, 222-224.

GinsBuRG, A. P. Is the illusory triangle physical or imaginary? Nature, 1975, 257, 219-220.

GREGORY, R. L. Cognitive contours. Nature, 1972, 238, 51-52.

Hohmann, A., \& Creutzfeldo, O. D. Squint and the development of binocularity in humans. Nature, 1975, 254, 613-614.

Julesz, B. Foundations of cyclopean perception. Chicago: University of Chicago Press, 1971.

Mitchell, D. E., Reardon, J., \& Muir, D. W. Interocular transfer of the motion aftereffect in normal and stereoblind humans. Experimental Brain Research, 1975, 22, 163-173.

MrtChell, D. E., \& W ARE, C. Interocular transfer of a visual aftereffect in normal and stereoblind humans. Journal of Physiology, 1974, 236, 707-721.
Movshon, J. A., Chambers, B. E. I., \& Blakemore, C. Interochlar transfer in normal vision, and those who lack stereopsis. Perception, 1972, 1, 483-490.

OVER, R. Comparison of normalization theory and neural enhancement explanation of negative aftereffects. Psychological Bulletin, $1971,75,225-243$.

Over, R., \& Lovegrove, W. Color-selectivity in simultaneous motion contrast. Perception \& Psychophysics, 1973, 14, 445-448.

Schumann, F. Einige Beobachtungen uber die Zusammenfassung von Gesichtseindrücken zu Einheiten. Psychologische Studien, $1904,1,1-32$

SeKULER, R.,\& GaNz, L. Aftereffect of seen motion with stabilized retinal image. Science, 1963, 139, 419-420.

SMITH, A., \& OvER, R. Tilt aftereffects with subjective contours. Nature, 1975, 257, 581-582.

Sмrtн, A. T., \& Over, R. Orientation masking and the tilt illusion with subjective contours. Perception, 1977, 6, 441-447.

Sutherland, N. S. Figural aftereffects and apparent size. Quarterly Journal of Experimental Psychology, 1961, 13, 222-228.

TYNAN, P., \& SEKUlER, R. Moving visual phantom: A new contour completion effect. Science, 1975, 188, 951-952.

Weisstein, N., Maguire, W., \& Berbaum, K. A phantommotion aftereffect. Science, 1977, 198, 955-958.

(Received for publication July 13, 1978; revision accepted November $13,1978$. ) 